Bond University

Research Repository

\title{
The nutrition impact symptoms (NIS) score detects malnutrition risk in patients admitted to nephrology wards
}

Maclaughlin, H. L.; Twomey, J.; Saunt, R.; Blain, S.; Campbell, Katrina L; Emery, P.

Published in:

Journal of Human Nutrition and Dietetics

DOI:

10.1111/jhn.12553

Licence:

Unspecified

Link to output in Bond University research repository.

Recommended citation(APA):

Maclaughlin, H. L., Twomey, J., Saunt, R., Blain, S., Campbell, K. L., \& Emery, P. (2018). The nutrition impact symptoms (NIS) score detects malnutrition risk in patients admitted to nephrology wards. Journal of Human Nutrition and Dietetics, 31(5), 683-688. https://doi.org/10.1111/jhn.12553

\section{General rights}

Copyright and moral rights for the publications made accessible in the public portal are retained by the authors and/or other copyright owners and it is a condition of accessing publications that users recognise and abide by the legal requirements associated with these rights.

For more information, or if you believe that this document breaches copyright, please contact the Bond University research repository coordinator 
The Nutrition Impact Symptoms (NIS) score detects malnutrition risk in patients admitted to nephrology wards.

\section{Abstract}

Background: Nutritional screening tools recommended for the general hospitalised population do not always adequately detect malnutrition risk in patients with kidney disease. This study assessed the validity and reliability of the Nutrition Impact Symptoms (NIS) score as a nutrition screening tool for hospitalised inpatients in nephrology wards.

Methods: Nutritional status was classified using Subjective Global Assessment (SGA). NIS scores were calculated from the total score of responses to questions assessing symptoms impacting upon nutritional status from the patient-generated SGA. Concurrent validity of NIS score was assessed using a receiver operating characteristics curve to predict malnutrition risk against SGA. Predictive validity was examined against length of hospital stay (LOS) and 30-day readmission using Poisson and logistic regression respectively. Inter-rater reliability of NIS scoring between assessors was determined using intra-class correlation.

Results: In 143 patients (90M; mean (SD) age 57.8 (15.8) years), malnutrition prevalence was 38\% (54/143) using SGA (rating B/C). Predicting malnutrition risk with an NIS score of $\geq 3$ had a sensitivity of 0.89 and a specificity of 0.65 (area under the curve $=0.81$ [95\% confidence interval (CI), $0.74-0.88]$ ). For each 1-point increase in NIS score, the model predicted a $1.9 \%$ rise in the risk of an increased LOS $(\mathrm{p}=0.002)$. 30-day readmission was not associated with NIS score. Inter-rater reliability was moderate (mean difference $=0.53$; intra-class correlation coefficient $=0.74 ; 95 \% \mathrm{CI} 0.57-0.85$ ).

Conclusions: NIS score is a valid stand-alone nutrition screening tool to identify malnutrition risk in nephrology inpatients, and is associated with length of hospital stay. 


\section{Introduction}

Malnutrition is a common complication of renal disease, particularly in the later stages of chronic kidney disease (CKD) (stages 3-5) (KDIGO, 2013). Studies demonstrate that over 50\% of patients admitted to nephrology wards are malnourished $(1,2)$, and uraemia, acidosis, dialysis and comorbidities all impact upon food intake and nutritional status in patients with kidney disease (3, 4). Impaired nutritional status is associated with poor clinical outcomes, including increased morbidity, longer hospital stay, readmission, reduced quality of life and poorer survival (5-9).

Nutritional screening is simple and efficient method of identifying those at risk of malnutrition, and screening in the hospital setting helps ensure that patients receive timely and effective treatment where needed $(10,11)$. In the UK, the Malnutrition Universal Screening Tool (MUST), identifies between 19$60 \%$ of hospitalised patients as at risk of malnutrition (10). However, evidence suggests that MUST lacks sensitivity and identifies only those at the highest malnutrition risk in patients with kidney disease when compared to nutrition assessment with Subjective Global Assessment (SGA) (12). Fluctuations in weight due to fluid retention masking undetected loss of tissue, may make detection or assessment of weight loss difficult; a factor which is essential for the accurate completion of MUST, and any other tool using BMI as a screening criteria (2). Other nutrition screening tools such as the Mini Nutrition Assessment and Malnutrition Screening Tool (MST) have also demonstrated little promise for use in patients with kidney damage (Afsar et al., 2006; Lawson et al., 2012). These findings are in agreement with the results of a systematic review reporting no single screening tool is appropriate for use in all hospitalised patients, and that future research should focus on trying to identify the most suitable screening tools for specific patient groups (13). Research on renal specific nutrition screening tools has continued. The renal nutrition screening toll (R-NST) was recently developed and tested for validity and feasibility (1). The R-NST demonstrated high sensitivity and specificity against SGA, however, when introduced into clinical practice there was low uptake when used by nurses, attributed to the need to access information from electronic clinical information systems, and poor agreement for scoring between researchers and nurses (1). Therefore, the need for a user friendly and valid nutrition screening tool for renal patients remains evident.

The Nutrition Impact Symptoms (NIS) score (Table 1) is part of the patient-generated SGA (PG-SGA), a validated nutrition assessment tool initially developed for use in oncology and also validated in haemodialysis $(14,15)$. Based on recent evidence supporting the efficacy of the NIS score as a nutrition screening tool for haemodialysis outpatients (16), and the high level of malnutrition with multiple aetiologies in patients admitted to nephrology wards, it is hypothesised that the NIS score may be a valid and reliable nutrition screening tool for renal inpatients on nephrology wards. 
66 A cross-sectional and observational validation study was conducted. Patients over 18 years were

67 considered eligible for inclusion in the study if they were admitted for a planned or unplanned/ emergency admission, under the care of a consultant nephrologist, to an acute renal unit consisting of two wards, and had been an inpatient for $<4$ days. All patients meeting the inclusion criteria were approached to participate in the study, during 3 separate recruitment periods of $4-8$ weeks, between July 2014 and April 2015, with different assessors for each period. Patients were introduced to the researcher by members of the clinical care team - either the patient's nurses, doctors or dietitian approached the patient to request permission for the researcher to inform the patient about the study. To maintain consistency, all researchers were trained in the study methods by the same trainer (HM), and reached competency standards for NIS score and SGA assessment prior to data collection. Patients were excluded if their total hospital stay was less than 24 hours, or were unable to provide informed consent.

Ethical approval was granted by the National Research Ethics Service Committee London - City \& East (reference number 15/LO/0204), and permission to use the PG-SGA (2015, v3.22.15; metric and non-metric version) was obtained from pt-global.org. Patients who met the inclusion criteria were given verbal and written information about the study prior to providing written informed consent. Confidentiality was maintained by coding patient identifiable details on paper records and in secure password protected electronic documentation.

\section{Concurrent Validity}

To calculate the NIS score, patients were asked "Have you had any of the following problems that have kept you from eating enough during the past two weeks?", followed by listing each NIS symptom in turn. The total NIS score was calculated by adding the scores for all symptoms identified positively by patients (Table 1). Individual NIS were only scored positively if they affected food intake (14). The SGA was completed at the same time using the standard method (12) and SGA global classifications were used to categorise nutritional status (A - well nourished, B - moderately malnourished and C severely malnourished). Patients identified as malnourished, with an SGA rating on B or C, were 
Table 1. Nutrition Impact Symptoms (NIS) score for symptoms impacting on food intake*. cases), of a range of NIS scores to detect malnutrition risk were determined.

The ability of the NIS score to identify malnutrition risk was assessed against the SGA global classification of nutritional status as the reference standard. Specificity (true negative cases / [(true negative + false positive] cases), and sensitivity (true positive cases / [true positive + false negative]

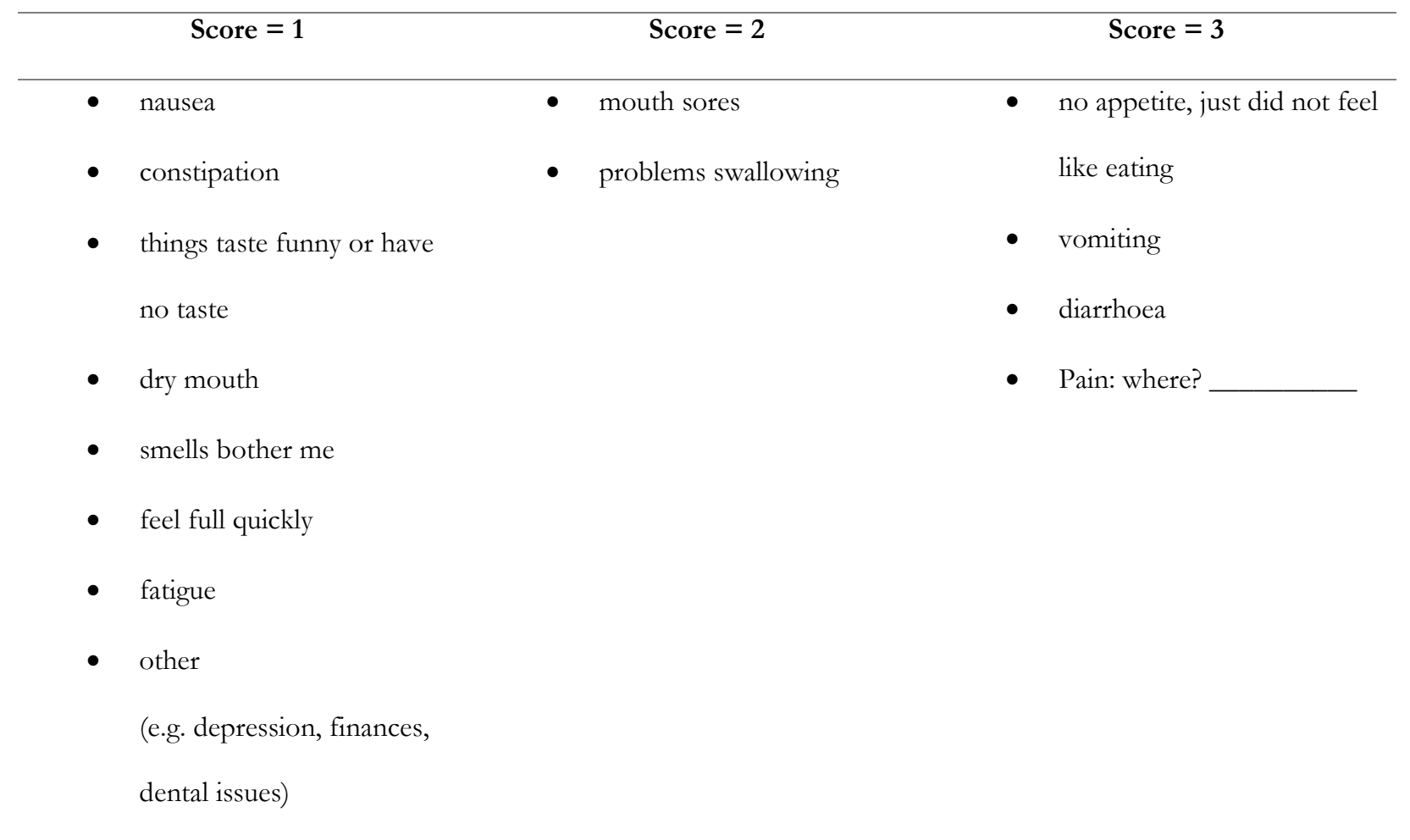

*Adapted from the PG-SGA (2015, v3.22.15; metric and non-metric version) http://pt-global.org

\section{Predictive and Clinical Validity}

Predictive validity was evaluated against length of stay (LOS) and readmission to any ward in the same hospital within 30 days of discharge. LOS was defined as the total number of days spent as an inpatient during the admission, calculated by subtracting the hospital admission date from the date of discharge. Serum albumin and C-reactive protein (CRP) concentrations on admission were recorded for each patient and the Charlson Comorbidity Index score (17) was calculated using clinical history and demographic data from electronic patient records.

\section{Inter-Rater Reliability}

The reliability of the NIS score was determined by repeating the NIS score in a subgroup of study participants ( $\mathrm{n}=43$ ) using a second measurer (a dietitian, nurse or healthcare assistant), blinded to the initial scoring, to assess NIS score only. The NIS score was repeated on the same day to ensure that conditions were comparable. 


\section{Data Analysis}

118 Statistical analysis was carried out using SPSS version 22 (IBM). Sample size calculations were based on findings from pilot testing of the NIS tool. With an expected prevalence of malnutrition at $50 \%$, $88 \%$ specificity and $80 \%$ sensitivity for NIS, and precision within $10 \%$ and type- 1 error of $5 \%, 125$ patients were required in the study. Normality of the data was assessed using histograms and the Shapiro-Wilk test of normality. Results were considered significant at $p<0.05$ and $95 \%$ confidence intervals (CIs) were computed where applicable. Baseline characteristics between malnourished (SGA B or C) and well nourished (SGA A) patients were compared with chi-squared tests - or Fisher's Exact test when needed - for categorical variables, and independent t-tests or Mann Whitney U tests for parametric and non-parametric continuous variables, respectively.

To establish the optimal NIS cut off score maximising the sensitivity and specificity of the tool in determining malnutrition risk, a receiver operating characteristic (ROC) curve and contingency table was produced comparing the NIS score with the SGA global rating of nutritional status as the reference standard (where SGA A = well-nourished and SGA B or C = malnourished). With the finalised risk categories, concurrent validity of the NIS score was examined against the SGA global rating of nutritional status to determine the sensitivity and specificity of the NIS score in identifying malnutrition risk using the contingency table. The associations between NIS score and clinical morbidity indicators, CRP, albumin and Charlson score, were assessed with Spearman rank correlations. The relationship between all 4 indicators and LOS or 30-day readmission were examined using Poisson linear regression analysis and forward, stepwise logistic regression analysis, respectively. Intra-class correlation tested the inter-rater reliability of the NIS score.

\section{Results}

Of the 178 potentially eligible patients, 143 patients were recruited to the study. Baseline characteristics are outlined in Table 2. 38\% of patients were malnourished when classified by SGA (33\% as SGA rating $\mathrm{B}$ and 5\% classified as SGA rating $\mathrm{C}$ ). Albumin, CRP and NIS score were significantly different between well-nourished and malnourished patients, and malnourished patients had a greater proportion of emergency/ unplanned admissions, compared to those who were well nourished.

\section{Concurrent Validity}

Examination of the contingency table indicated that the concurrent validity of the NIS score was greatest at a score of $\geq 3$, classifying $55 \%(79 / 143)$ of patients as at risk of malnutrition. The area under the ROC curve (AUC) was 0.81 (95\% CI 0.74 - 0.88), indicating good concurrent validity (13). 
151 Sensitivity was $89 \%$ (true risk of malnutrition identified) and specificity was $65 \%$ (true no risk of

152 malnutrition identified), compared to SGA.

153

154 Table 2. Baseline characteristics of patients admitted to acute renal inpatient wards by nutritional status

\begin{tabular}{|c|c|c|c|}
\hline \multirow[t]{2}{*}{ Variable } & Well-Nourished & Malnourished & \multirow[t]{2}{*}{$\mathrm{p}$} \\
\hline & $\mathrm{SGA}^{1} \mathbf{A}$ & SGA B or C & \\
\hline $\mathrm{N}(\%)$ & $89(62 \%)$ & $54(38 \%)$ & \\
\hline Age (years), mean $\pm \mathrm{SD}^{2}$ & $57.4 \pm 15.7$ & $58.3 \pm 16.0$ & $0.8^{8}$ \\
\hline \multicolumn{4}{|l|}{ Gender, n (\%) } \\
\hline Male & $55(62 \%)$ & $35(65 \%)$ & 0.7 \\
\hline Female & $34(38 \%)$ & $19(35 \%)$ & \\
\hline \multicolumn{4}{|l|}{ Ethnicity, n (\%) } \\
\hline White & $33(37 \%)$ & $27(50 \%)$ & 0.2 \\
\hline Black & $33(37 \%)$ & $12(22 \%)$ & \\
\hline Other & $23(26 \%)$ & $15(28 \%)$ & \\
\hline Albumin $(\mathrm{g} / \mathrm{L})$ mean $\pm \mathrm{SD}$ & $38.3 \pm 5.5$ & $35.7 \pm 6.3$ & $0.009^{8}$ \\
\hline $\mathrm{CRP}^{3}(\mathrm{mg} / \mathrm{L})$, median $(\mathrm{IQR})$ & $9.7(3.8-28.8)$ & $25.1(8.0-93.5)$ & $0.014^{7}$ \\
\hline Charlson Score, mean \pm SD & $5.1 \pm 2.3$ & $5.2 \pm 2.5$ & $0.6^{8}$ \\
\hline \multicolumn{4}{|l|}{ Admission type, n (\%) } \\
\hline Elective & $37(42 \%)$ & $11(20 \%)$ & 0.01 \\
\hline Unplanned & $52(58 \%)$ & $43(80 \%)$ & \\
\hline \multicolumn{4}{|l|}{ Kidney Damage, n (\%) } \\
\hline $\mathrm{CKD}^{4}$ stages $1-2$ & $7(8 \%)$ & $2(4 \%)$ & $0.2^{9}$ \\
\hline CKD stages $3-4$ & $22(60 \%)$ & $12(22 \%)$ & \\
\hline CKD stage 5 & $53(24 \%)$ & $30(56 \%)$ & \\
\hline Acute Kidney Injury & $7(8 \%)$ & $10(18 \%)$ & \\
\hline Length of stay (days), median & $4(2-8)$ & $5(3-11)$ & $0.1^{7}$ \\
\hline$(\mathrm{IQR})^{5}$ & $1.0(0-4)$ & $7.0(4-10)$ & $<0.001^{7}$ \\
\hline
\end{tabular}

155 1. SGA, Subjective Global Assessment; 2. SD, standard deviation; 3. CRP, C-reactive protein; 4. CKD, chronic kidney

156 disease; 5. IQR, interquartile range; 6. NIS, Nutrition Impact Symptoms; 7. Mann Whitney U test; 8. Independent t-test; 9.

157 Fisher's Exact test. 


\section{Predictive and Clinical Validity}

160 Using rank correlation, NIS score was associated with CRP $(\rho=0.22, \mathrm{p}=0.011)$, but not albumin or

161 Charlson score. In the Poisson regression model, all factors predicted an increased risk of longer LOS;

162

\begin{tabular}{lccc}
\hline Predictor variables & Regression co-efficient ( $\beta)$ & $\begin{array}{c}\text { Incident rate ratio } \\
\left(\mathbf{e}^{\boldsymbol{\beta}}\right) \text { and } \mathbf{9 5} \% \\
\end{array}$ & \\
& & $0.93(0.92-0.94)$ & $<0.001$ \\
& & $1.002(1.001-1.002)$ & $<0.001$ \\
\hline Albumin concentration (g/L) & -0.72 & $1.019(1.007-1.031)$ & 0.002 \\
C reactive protein (mg/L) & 0.002 & $0.95(0.93-0.98)$ & $<0.001$ \\
Nutrition Impact Symptoms (NIS) score & 0.019 & & \\
Charlson Comorbidity Index & -0.05 & & \\
\hline
\end{tabular}

172

Table 4 - Multivariable logistic regression for factors related to hospital readmission within 30 days

\begin{tabular}{lccc}
\hline Predictor variables & Odds ratio ( $\beta)$ & $\mathbf{9 5 \%}$ Confidence Interval & $\mathbf{p}$ \\
\hline Unplanned admission & 4.97 & $1.36-18.05$ & 0.02 \\
Serum albumin & 1.09 & $1.01-1.19$ & 0.04 \\
Length of stay (LOS) & 1.05 & $1.01-1.09$ & 0.02 \\
\hline
\end{tabular}

\section{Inter-Rater Reliability}

176 There was no difference in the total NIS score between measurers in $37 \%$ of cases. The mean

177 difference between repeated NIS scores was $0.53 \pm 2.81$ (mean \pm SD). The Intra-class correlation 178 coefficient was 0.74 (95\% CI 0.57 - 0.85), indicating moderate reliability between users. 


\section{Discussion}

181 This study established that the NIS score is a valid nutrition screening tool to assess malnutrition risk in patients admitted to nephrology wards. Concurrent, clinical and predictive validity were demonstrated through comparison to the SGA global rating of nutritional status, and by association with CRP, and increased risk of longer LOS, respectively.

Compared with SGA, the concurrent validity was deemed to be at its highest with an NIS score of $\geq 3$.

This NIS score cut-off is higher than that selected in a previous investigation involving 213 haemodialysis outpatients, which found that a NIS score of $\geq 2$ was most effective at detecting malnutrition risk (16). Median NIS scores in the well-nourished and malnourished groups were 1.0 and 3.5 points lower, respectively, than the median scores in the present study, and less than a quarter of patients were classified as malnourished, indicating that when malnutrition risk is lower, the threshold for detection with a nutrition screening tool is also lower, in order to maximise sensitivity. Together, these studies demonstrate the flexibility of the NIS score as a screening tool across different setting in patients with CKD, and also the importance of validating nutrition screening tools within the intended patient population.

A nutrition screening tool should have a high level of sensitivity to detect malnutrition, to reduce the risk of failing to detect malnutrition risk in a malnourished patient (false negative result) (10). An NIS score $\geq 3$ had a sensitivity of $89 \%$, demonstrating a far superior ability to detect malnutrition risk in patients with kidney disease than the MUST and MST tools; which were shown to have sensitivities of $54 \%$ and $49 \%$ compared to SGA respectively (2). With a specificity of $65 \%$, the NIS score at a cut-off of $\geq 3$ was relatively effective at identifying well-nourished patients, with similar specificity to the MST (18), although it does carry a reasonably high rate of false positive results. More recently, another nutrition screening tool, the R-NST was developed specifically for renal inpatients (1). The R-NST is more complex than the NIS score, and includes biochemical parameters alongside nutrition impact symptoms and weight loss history. The R-NST demonstrated high sensitivity (97\%) and moderately high specificity (74.4\%), compared to SGA. However in the reliability and feasibility phase of the study, the R-NST tool had low levels of completion by clinical staff due to the time taken to calculate 6month weight change, and extract the clinical data from the electronic medical records. Reliability was difficult to measure due to the very low completion rate for nursing staff (1), Together these results indicate that the R-NST may have limited translational capacity for use in clinical practice. 
This study is the first investigation to show that the NIS score is associated with LOS, an indicator of morbidity in patients with kidney disease (19), thus demonstrating a degree of predictive validity of the NIS score. The NIS score has also previously been shown to predict long term clinical outcomes in patients on maintenance haemodialysis, as an NIS score of $\geq 2$ was associated with a higher risk of mortality, whereas the SGA global rating was not (16).

The NIS score had moderate inter-rater reliability, with an ICC of 0.74 , and identical NIS scores were reported between assessors in $37 \%$ of patients. Whilst this is less than ideal, it is significantly higher than the agreement between assessors using the R-NST, where there same score was achieved in the repeated measure in only $8 \%$ of cases (1). Reliability of nutritional assessment using SGA can also be limited, with only fair inter-rater reliability between assessors following completion of an online training package (20). The research team provided brief training to clinical assessors before determining the NIS score. Between-user differences might be minimised by introducing more in-depth training for all assessors, where measurers would be expected to demonstrate competency before using the NIS score in practice.

The limitations of this study are also acknowledged. In each assessment, the NIS and the SGA were undertaken by the same researcher in a single session, so blinding the researchers to the outcomes of the individual components was not possible. However, as the study was conducted in three different time periods with different researchers, the robustness of the tool across users and over time is demonstrated. There are several advantages of using the NIS score as a nutrition screening tool in preference to other tools. The NIS score does not require measurement of body weight, knowledge of oedema free weight or previous weight loss. The NIS score also has no biochemical parameters included, so it can be quickly and easily completed at the bedside. Furthermore, the NIS score can identify the main factors impacting on food intake early during hospitalisation and can thus inform subsequent interventions to improve nutritional status (16). As the NIS score identifies specific factors relating to malnutrition risk, it guides the choice of clinical intervention. Symptoms such as dry mouth, taste changes, nausea, vomiting and constipation can all be treated clinically, whilst swallowing problems, feeling full quickly and fatigue require specific nutritional interventions.

\section{The outcomes of this study support the use of the NIS score as a nutrition screening tool for} hospitalised patients on nephrology wards, adding to previous findings supporting its use in haemodialysis outpatients. Concurrent, predictive and clinical validity were demonstrated against the SGA global rating of nutritional status, and the reliability between users was moderate. Future research into the use of the NIS as a nutrition screening tool should focus on the effect of training and nursing 
involvement in clinical implementation and the effect on longer-term clinical outcomes such as mortality, and patient focused outcomes such as quality of life, and discharge with maintained or improved functional capacity.

\section{Transparency Declaration}

The lead author affirms that this manuscript is an honest, accurate, and transparent account of the study being reported. The lead author affirms that no important aspects of the study have been omitted and that any discrepancies from the study as planned have been explained.

\section{References}

1. Xia YA, Healy A, Kruger R. Developing and Validating a Renal Nutrition Screening Tool to Effectively Identify Undernutrition Risk Among Renal Inpatients. J Ren Nutr. 2016;26(5):299-307.

2. Lawson CS, Campbell KL, Dimakopoulos I, Dockrell ME. Assessing the validity and reliability of the MUST and MST nutrition screening tools in renal inpatients. Journal of renal nutrition : the official journal of the Council on Renal Nutrition of the National Kidney Foundation. 2012;22(5):499-506.

3. K/DOQI National Kidney Foundation. Clinical practice guidelines for chronic kidney disease: evaluation, classification, and stratification. American Journal of Kidney Diseases. 2002;39(2 Suppl 1):S1-266.

4. K/DOQI National Kidney Foundation. Clinical practice guidelines for nutrition in chronic renal failure. . American Journal of Kidney Diseases 2000;35(6 Suppl 2):S1-140.

5. Agarwal E, Ferguson M, Banks M, Batterham M, Bauer J, Capra S, et al. Malnutrition and poor food intake are associated with prolonged hospital stay, frequent readmissions, and greater in-hospital mortality: Results from the Nutrition Care Day Survey 2010. Clin Nutr. 2013;32(5):737-45.

6. de Mutsert R, Grootendorst DC, Boeschoten EW, Brandts H, van Manen JG, Krediet RT, et al. Subjective global assessment of nutritional status is strongly associated with mortality in chronic dialysis patients. The American Journal of Clinical Nutrition. 2009;89(3):787-93.

7. Campbell KL, Ash S, Bauer JD. The impact of nutrition intervention on quality of life in pre-dialysis chronic kidney disease patients. Clin Nutr. 2008;27(4):537-44.

8. Ikizler TA, Wingard RL, Harvell J, Shyr Y, Hakim RM. Association of morbidity with markers of nutrition and inflammation in chronic hemodialysis patients: a prospective study. Kidney Int. 1999;55(5):1945-51.

9. Kaysen GA, Muller HG, Young BS, Leng X, Chertow GM. The influence of patient- and facility-specific factors on nutritional status and survival in hemodialysis. J Ren Nutr. 2004;14(2):72-81.

10. Stratton RJ, Hackston A, Longmore D, Dixon R, Price S, Stroud M, et al. Malnutrition in hospital outpatients and inpatients: prevalence, concurrent validity and ease of use of the 'malnutrition universal screening tool' ('MUST') for adults. Br J Nutr. 2004;92(5):799-808.

11. National Institute for Health and Clinical Excellence. Nutrition Support in Adults Quality Standard (QS24). 2012.

12. Detsky AS, Mclaughlin JR, Baker JP, Johnston N, Whittaker S, Mendelson RA, et al. What Is Subjective Global Assessment of Nutritional-Status. Jpen-Parenter Enter. 1987;11(1):8-13.

13. van Bokhorst-de van der Schueren MA, Guaitoli PR, Jansma EP, de Vet HC. Nutrition screening tools: does one size fit all? A systematic review of screening tools for the hospital setting. Clin Nutr. 2014;33(1):39-58.

14. Ottery FD. Definition of standardized nutritional assessment and interventional pathways in oncology. Nutrition. 1996;12(1 Suppl):S15-9.

15. Desbrow B, Bauer J, Blum C, Kandasamy A, McDonald A, Montgomery K. Assessment of nutritional status in hemodialysis patients using patient-generated subjective global assessment. J Ren Nutr. 2005;15(2):211-6. 
16. Campbell KL, Bauer JD, Ikehiro A, Johnson DW. Role of Nutrition Impact Symptoms in Predicting Nutritional Status and Clinical Outcome in Hemodialysis Patients: A Potential Screening Tool. Journal of Renal Nutrition. 2013;23:302-7.

17. Charlson ME, Pompei P, Ales KL, MacKenzie CR. A new method of classifying prognostic comorbidity in longitudinal studies: development and validation. J Chronic Dis. 1987;40(5):373-83.

18. Ferguson M, Capra S, Bauer J, Banks M. Development of a valid and reliable malnutrition screening tool for adult acute hospital patients. Nutrition. 1999;15(6):458-64.

19. Pupim LB, Evanson JA, Hakim RM, Ikizler TA. The extent of uremic malnutrition at the time of initiation of maintenance hemodialysis is associated with subsequent hospitalization. J Ren Nutr. 2003;13(4):259-66.

20. Steiber A, Leon JB, Secker D, McCarthy M, McCann L, Serra M, et al. Multicenter study of the validity and reliability of subjective global assessment in the hemodialysis population. Journal of Renal Nutrition. 2007;17(5):336-42. 